\title{
REPRESENTAÇÕES SOCIAIS DE TRABALHADORES DA ATENÇÃO BÁSICA DE SAÚDE SOBRE ENVELHECIMENTO
}

\author{
Cristina Katya Torres Teixeira MENDES ${ }^{a}$, Maria do Socorro Costa Feitosa ALVES ${ }^{b}$, Antonia Oliveira SILVAc, \\ Maria Adelaide Silva PAREDES ${ }^{d}$, Tatyanni Peixoto RODRIGUES
}

\section{RESUMO}

Este estudo teve o objetivo de conhecer as representações sociais sobre envelhecimento construídas por trabalhadores de saúde da Atenção Básica. Trata-se de um estudo exploratório, com a participação de 204 trabalhadores da Atenção Básica em Saúde, do município de João Pessoa, Paraíba. Para coleta de dados, foi utilizada uma entrevista semiestruturada. Os dados obtidos das 204 entrevistas foram analisados com o auxílio do programa informático Alceste, versão 2010. Os resultados apontaram cinco classes ou categorias: visão sobre envelhecimento; dimensões psicossociais; tempo de dúvidas; envelhecimento como processo; envelhecimento versus doença, com conteúdos tanto positivos: alegria, atenção, filhos, aposentadoria, cuidador, direitos, maturidade e sabedoria, quanto negativos: deficiências, decadência, esquecimento, fragilidade, limitação, rugas, dependência e doença. Observou-se que os sentidos associados ao envelhecimento mostra a necessidade de um cuidar integral e humanizado à pessoa idosa.

Descritores: Trabalhadores. Saúde. Envelhecimento. Enfermagem.

\section{RESUMEN}

Este estudio tuvo por objetivo de conocer las representaciones sociales del envejecimiento construidas por los trabajadores de salud de la Atención Primaria. Es un estudio exploratorio con la participación de 204 trabajadores de la ciudad de João Pessoa, Paraíba. Para la recogida de datos se utilizó una entrevista semiestructurada. Los datos obtenidos de las 204 entrevistas fueron analizados con de la ayuda de software Alceste versión 2010. Los resultados mostraron cinco clases o categorías: visión sobre el envejecimiento, las dimensiones psicosociales, tiempo de dudas, el envejecimiento como un proceso, el envejecimiento versus la enfermedad. Los contenidos son tanto positivos: la alegría, la atención, los niños, la jubilación, los derechos de cuidador, la madurez y la sabiduría, como negativos: deficiencias, la decadencia, el abandono, la debilidad, la limitación, las arrugas, la adicción y la enfermedad. Hemos observado los sentidos asociados con el envejecimiento la necesidad de un cuidar pleno y humanizado a los ancianos.

Descriptores: Trabajadores. Salud. Envejecimiento. Enfermería.

Título: Representación de los trabajadores sociales de la salud de atención primaria sobre el envejecimiento.

\section{ABSTRACT}

The objective of this study was to get to know the social representations on aging developed by Primary Care health workers. This is an exploratory study involving 204 Primary Health Care workers, in the city of João Pessoa, in the state of Paraíba. For data collection we used a semi-structured interview. The data obtained from 204 interviews was analyzed with the help of the Alceste software version 2010. The results indicated five classes or categories: vision of aging, psychosocial dimensions, a time of doubts, aging as a process, and aging versus disease, with positive content: joy, care, children, retirement, caregiver rights, maturity and wisdom, as well as negative factors: impairments, decadence, neglect, fragility, limitation, wrinkles, dependency and disease. It was observed that these meanings associated with aging express the need for total and humanized elderly care.

Descriptors: Workers. Health. Aging. Nursing.

Title: Social representations on aging by primary care health workers.

a Fisioterapeuta, Mestre em Enfermagem, Doutoranda do Programa de Pós-Graduação em Ciências da Saúde da Universidade Federal do Rio Grande do Norte, Natal, Rio Grande do Norte, Brasil. Pesquisadora do Grupo Internacional de Estudos e Pesquisas em Envelhecimento e Representações Sociais.

b Odontóloga, Prof.PhD do Departamento de Odontologia e Docente do Programa de Pós-Graduação em Ciências da Saúde da Universidade Federal do Rio Grande do Norte, Natal, Rio Grande do Norte, Brasil. Pesquisadora do Grupo Internacional de Estudos e Pesquisas em Envelhecimento e Representações Sociais.

c Enfermeira, Prof.PhD do Departamento de Saúde Pública e Psiquiatria e Coordenadora-Docente do Programa de Pós-Graduação em Enfermagem da Universidade Federal da Paraíba, João Pessoa, Paraíba, Brasil. Líder do Grupo Internacional de Estudos e Pesquisas em Envelhecimento e Representações Sociais.

d Fisioterapeuta, Mestre em Engenharia de Produção, Doutora em Ciências da Saúde, Pós-Doutorando em Enfermagem do Programa de Pós-Graduação em Enfermagem da Universidade Federal da Paraíba, João Pessoa, Paraíba, Brasil. Pesquisadora do Grupo Internacional de Estudos e Pesquisas em Envelhecimento e Representações Sociais.

e Enfermeira, Mestranda do Programa de Pós-Graduação em Enfermagem da Universidade Federal da Paraíba, João Pessoa, Paraíba, Brasil. Estudante do Grupo Internacional de Estudos e Pesquisas em Envelhecimento e Representações Sociais. 


\section{INTRODUÇÃO}

O envelhecer é um fenômeno natural da vida de um indivíduo, ou seja, é um processo geral, dinâmico, progressivo, lento e gradual, para o qual concorre uma multiplicidade de fatores genéticos, biológicos, sociais, ambientais, psicológicos e culturais característicos da pessoa e a pressão do meio ambiente. Seus efeitos são dependentes da capacidade de substituição e de compensação da pessoa e das exigências do meio $^{(1)}$.

Do ponto de vista demográfico e do plano individual envelhecer significa aumentar o número de anos vividos ${ }^{(1)}$. Paralela à evolução cronológica, coexistem fenômenos de natureza biopsíquica e social, importantes para a percepção da idade e do envelhecimento.

O envelhecimento nas sociedades desenvolvidas é o resultado da melhoria das condições de vida e dos progressos da ciência e da medicina que têm trazido um aumento significativo dos índices de longevidade expresso na esperança média de vida que atinge números nunca antes registrados, mesmo com a drástica redução da mortalidade infantil, com a diminuição das taxas de fecundidade, aliada à maior longevidade, tem provocado uma profunda alteração na geometria das pirâmides populacionais ${ }^{(2)}$.

O mundo está a envelhecer, em que em todo o mundo estima-se que o número de pessoas com 60 e mais anos conheça um aumento na ordem de $85 \%$. Com relação ao crescimento da população de idosos vem se observando que este ocorre de forma mais acentuada nos países em desenvolvimento, embora este contingente ainda seja proporcionalmente bem inferior ao encontrado nos países desenvolvidos. Em relação aos países da América Latina, o Brasil assume uma posição intermediária com uma população de idosos correspondendo a $8,6 \%$ da população total ${ }^{(3)}$.

Esse impacto marcará, sem dúvida, o processo de desenvolvimento econômico e social e representa um grande desafio em termos de sustentabilidade, quer do sistema de segurança social, quer do próprio modelo produtivo, mas como um repensar a ser feito no âmbito do respeito integral da pessoa humana em todas as suas dimensões ${ }^{(2)}$, gerando grandes desafios para essas sociedades, que precisam ser capazes de promover o envelhecimento saudável e ativo ${ }^{(4)}$.
O conceito de envelhecimento bem-sucedido envolve baixo risco de doenças e de incapacidades, funcionamento físico e mental excelente e envolvimento ativo com a vida. Depende da capacidade de adaptação às mudanças físicas, emocionais e sociais. Esta habilidade é o resultado da estrutura psicológica e de condições sociais construídas ao longo da vida ${ }^{(5)}$.

É importante se conhecer o que pensam os profissionais de saúde sobre envelhecimento para se trabalhar com aspectos específicos à saúde da pessoa idosa capaz de auxiliar na otimização do suporte familiar e comunitário, assim como no fortalecimento de vínculos de co-responsabilidade.

Enquanto fenômenos sempre ativados, as representações sociais constituem formas de conhecimentos/informações construídas e/ou atualizadas na vida social das pessoas. $\mathrm{O}$ estudo de tais fenômenos possibilita a apreensão de diferentes aspectos subjetivos envolvidos no cotidiano da organização de um saber próprio de uma realidade social. $\mathrm{O}$ conjunto desses componentes em processo configura uma totalidade significante que, em relação com a ação $0^{(5)}$.

Assim sendo, nas sociedades contemporâneas, em particular nas sociedades dos países desenvolvidos, o envelhecimento das populações tem ganhado uma dimensão e uma complexidade ${ }^{(2)}$, assim como, nos países em desenvolvimento, ratificam profundas modificações do ponto de vista das implicações relacionadas com os cuidados de saúde, a organização social e familiar e da parte dos governos das mudanças das condições concretas de saúde e de vida, considerando além desses aspectos as dimensões subjetivas próprias do envelhecimento. Logo, este estudo tem o objetivo de conhecer as representações sociais sobre envelhecimento construídas por trabalhadores de saúde da Atenção Básica.

\section{METODOLOGIA}

Trata-se de um recorte extraído da Tese de Doutorado $^{(6)}$ do Programa de Pós-Graduação em Ciências da Saúde da Universidade Federal do Rio Grande do Norte - RN, inserida na Pesquisa sobre Tecnologias Assistivas para Idosos Atendidos em Unidades de Saúde da Família no município de João Pessoa, Paraíba, financiada pelo Ministério 
da Saúde em convênio com o Programa de Pós-Graduação de Enfermagem da Universidade Federal da Paraíba, subsidiada no aporte teórico das representações sociais ${ }^{(7)}$.

Participaram do estudo 204 (duzentos e quatro) profissionais de saúde, que trabalham nas Unidades de Saúde, escolhidos de forma aleatória, de ambos os sexos, que aceitaram participar do estudo, atendendo as recomendações previstas na Resolução 196/1996 $6^{(8)}$, do Ministério da Saúde, sobre pesquisa envolvendo seres humanos, após aprovação pelo comitê de ética em pesquisa do Hospital Universitário Lauro Wanderley/UFPB (Protocolo CEP/HULW $n^{\circ} .261 / 09$, fr: 294027).

A coleta de dados foi realizada a partir do Teste da Associação Livre de Palavras, formado na primeira por uma questão única, com o termo indutor «envelhecimento» e a segunda, contemplou as variáveis: sexo, idade e tipo de profissional.

Os dados empíricos coletados a partir das respostas evocadas pelos profissionais foram organizados em um banco de dados que foi sumetido a uma análise de conteúdo textual com o auxílio do programa informático Alceste (Análise Lexical Contextual de um Conjunto de Segmentos de Texto) versão 2010, que utiliza a linguagem escrita ou transcrita, como: material escrito da mídia; falas de entrevistas; depoimentos; relatos; documentos e textos produzidos de diferentes técnicas para coleta. Este programa realiza uma análise de classificação hierárquica descendente e lexicográfica do material textual, oferecendo contextos ou classes/categorias que são caracterizados pelo seu vocabulário e pelos segmentos de textos que compartilham do vocabulário $^{(9)}$.

Esse procedimento possibilitou para o estímulo envelhecimento o delineamento de cinco classes em função da ocorrência e co-ocorrência das palavras contidas no vocabulário e da sua contextualização dentro dos segmentos de textos articulados para um corpus formado por 204 Unidades de Contextos Iniciais (UCI's) ou 204 testes da Associação Livre de Palavras, correspondente as entrevistas, com um aproveitamento de $82,84 \%$ do corpus submetido ao Alceste após análise, posteriormente foi interpretado a luz da teoria das representações sociais.

\section{RESULTADOS E DISCUSSÃO}

Participaram do estudo 178 mulheres $(87,25$ $\%)$ e 26 homens (12,75\%), que trabalham nas Unidades de Saúde da Família do município de João Pessoa, em sua maioria estão na faixa etária entre $40-49$ anos de idade (28,92\%), e possuem curso superior $(81,86 \%)$ (Tabela 1$)$.

Tabela 1 - Distribuição dos sujeitos do estudo. João Pessoa, PB, 2011.

\begin{tabular}{lcc}
\hline \multicolumn{1}{c}{ Variáveis } & Perfil dos sujeitos do estudo \\
\hline Sexo & $\mathbf{n}$ & $\%$ \\
Feminino & 178 & \\
Masculino & 26 & 87,25 \\
\hline Idade & & 12,75 \\
20 a 29 anos & 45 & 22,05 \\
30 a 99 anos & 56 & 27,45 \\
40 a 49 anos & 59 & 28,92 \\
50 a 59 anos & 31 & 15,2 \\
60 e mais & 09 & 4,41 \\
O6 não respondeu & 04 & 1,97 \\
\hline Profissionais & & \\
Nível Superior & 167 & 81,86 \\
Nível Médio & 25 & 12,25 \\
Nível Fundamental & 12 & 5,89 \\
\hline
\end{tabular}

Fonte: Pesquisa do MS, 2011. 
As representações sociais sobre envelhecimento elaboradas pelos 204 trabalhadores definidas a partir de um corpus constituído por 206 palavras diferentes, com frequência igual ou inferior a quatro, responsáveis pela formação das cinco classes ou categorias semânticas: a) visão sobre envelhecimento; b) dimensões psicossociais; c) tempo de dúvidas; d) envelhecimento como processo; e) envelhecimento versus doença. Para essas pesquisa considerou-se para interpretação as palavras com frequência mínima correspondente a dez.

Visão sobre envelhecimento (classe 1) e as dimensões biopsicossociais do envelhecimento (classe 2).

Essas duas classes apresentam a visão dos profissionais sobre o envelhecimento e as dimensões biopsicossociais do envelhecimento, respectivamente com conteúdos positivos e negativos em que os profissionais atribuem dimensões psicossociais para falarem sobre envelhecimento no olhar dos profissionais de saúde (Tabela 2).

A tabela 2, apresenta as duas primeiras classes (1 e 2) em que na classe 1 - visão sobre o envelhecimento, em que se pode identificar uma visão positiva dos trabalhadores ao associarem ao envelhecimento dimensões como: alegria, atenção, filhos, aposentadoria, cuidador, direitos, maturidade e sabedoria; enquanto a visão negativa é representada por: decadência, esquecimento, fragilidade, limitação e rugas. Essas mostram características referentes ao status tanto positivo como negativo das pessoas idosas em quatro áreas: biológica, psíquica, sociológica e econômica ${ }^{(10)}$. Na classe 2 , dimensões biopsicossociais do envelhecimento, os profissionais atribuem ao envelhecimento sentimentos de: abandono, angústia, desprezo, dor, solidão e tristeza (Tabela 2).

Verificou-se que os profissionais de saúde conhecem as síndromes geriátricas, e os múltiplos problemas e a importância do processo de envelhecimento com ênfase no conhecimento da rede de suporte social indisponível.

As alterações funcionais próprias do envelhecimento associadas à maior prevalência de doenças crônicas que podem levar à deterioração da habilidade de manutenção da independência ${ }^{(11)}$.

Tempo de dúvidas (classe 3) e envelhecimento enquanto processo (classe 4).

Tabela 2 - Palavras associadas significativamente às classes 1 e 2. João Pessoa, PB, 2011

CLASSE 1 Visão sobre envelhecimento
CLASSE 2

Dimensões biopsicossociais do envelhecimento

\begin{tabular}{llll}
\hline Palavras & F & Palavras & F \\
\hline Alegria & 10 & & 10 \\
Aposentadoria & 44 & Abandono & 10 \\
Atenção & 14 & Angústia & 10 \\
Cuidador & 46 & Asilo & 24 \\
Decadência & 10 & Cabelos Brancos & 10 \\
Direitos & 10 & Ciclo da Vida & 10 \\
Esquecimento & 24 & Depressão & 12 \\
Fase & 16 & Desprezo & 150 \\
Filhos & 18 & Doença & 30 \\
Fragilidade & 18 & Dor & 10 \\
Idade & 12 & Respeito & 38 \\
Inevitável & 16 & Saudade & 58 \\
Limitação & 30 & Solidão & 36 \\
Maturidade & 20 & Tristeza & 20 \\
Rugas & 34 & Viver & \\
Sabedoria & 42 & & \\
\hline
\end{tabular}


Tabela 3 - Palavras associadas significativamente às classes 3 e 4. João Pessoa, PB, 2011

\begin{tabular}{lrllc}
\hline & $\begin{array}{c}\text { CLASSE 3 } \\
\text { Tempo de dúvidas }\end{array}$ & \multicolumn{2}{c}{$\begin{array}{c}\text { CLASSE 4 } \\
\text { Envelhecimento como processo }\end{array}$} \\
\hline \multicolumn{1}{c}{ Palavras } & F & & \multicolumn{2}{c}{ Palavras } \\
\hline Acompanhamento & 12 & Adaptação & 10 \\
Convivência & 16 & Experiência & 86 \\
Dúvida & 16 & Idoso & 26 \\
Família & 34 & Interação & 08 \\
Filhos & 18 & Maturidade & 20 \\
Netos & 24 & Modificações & 12 \\
Obstáculos & 14 & Processo & 10 \\
Tempo & 16 & Qualidade de Vida & 18 \\
& & & Saúde & 48 \\
\hline
\end{tabular}

Fonte: Pesquisa do MS, 2011.

Na classe três, tempo de dúvida, se destacam conteúdos significativos como: acompanhamento, convivência, dúvida, obstáculos e tempo, como um determinante do futuro da pessoa que entra no processo do envelhecimento (Tabela 3).

Este olhar reflete uma forma de avaliação do envelhecimento frente ao idoso associadas às relações familiares confirmadas nas falas dos profissionais como, família, netos e filhos. Essa classe ainda nos mostra a capacidade que os profissionais de saúde têm de identificar os fatores determinantes da fragilidade e da qualidade de vida da pessoa idosa, em seu contexto familiar e social, bem como compreender o sentido da responsabilização compartilhada como base para o desenvolvimento das ações que contribuem para o alcance de uma vida saudável ${ }^{(12)}$.

O envelhecimento enquanto processo - classe quatro (Tabela 3) é visto pelos profissionais de saúde como modificações, adaptação e amadurecimento. Para além das alterações visíveis no corpo, o envelhecimento proporciona ao ser humano uma série de mudanças psíquicas, que pode resultar na dificuldade de se adaptar a novos papéis; falta de motivação e de dificuldade de planear o futuro; necessidade de trabalhar as perdas orgânicas, afetivas, e sociais; dificuldade de se adaptar a mudanças rápidas, que podem ser dramáticas; alterações psíquicas que requerem tratamento especializado; imagens negativas e baixa autoestima ${ }^{(11)}$.

É necessário deixar claro que mesmo o idoso portador de doença poderá sentir-se saudável, ativo
Tabela 4-Palavras associadas significativamente à classe 5. João Pessoa, PB, 2011

\begin{tabular}{lc}
\hline \multicolumn{2}{c}{ CLASSE 5 } \\
Envelhecimento versus doença \\
\hline Palavras
\end{tabular}

Fonte: Pesquisa do MS, 2011.

em seu meio e ter boa qualidade de vida, uma vez que para tanto, a sua manutenção está intimamente vinculada a autonomia e independência, que são bons indicadores de saúde para a população idosa ${ }^{(12)}$.

Envelhecimento versus doença (Classe 5)

Os sentidos associados ao envelhecimento pelos trabalhadores de saúde versus doença (Tabela 4).

Eles descrevem por um lado, aspectos associados às dimensões que impedem o envelhecimento ativo como: limitação bengala e dependência; por outro, apontam a doença capaz de leva-los a solidão. Tais aspectos são informações importantes sugestivas de inclusão nas ações para atendimento a pessoa idosa nos trabalhos em grupo e nas atividades profissionais e práticas de saúde. 
Observa-se nas falas dos profissionais uma ênfase para os aspectos relacionados com o idoso em que é percebido como portador de uma diminuição da capacidade funcional, retratadas nos elementos já mencionados, como: cansaço físico, células morrendo, limitação e solidão mostrando assim a importância da atividade física para a prevenção e promoção da saúde ${ }^{(13)}$.

O envelhecimento no olhar dos profissionais de saúde aponta dimensões subjetivas importantes na constituição das representações sociais, com conteúdos positivos e negativos.

Os sentidos associados ao envelhecimento são objetivados em imagens ou no campo de representação dimensionadas nas cinco classes ou categorias contemplando imagens positivas e negativas, além de um posicionamento favorável e desfavorável sobre o envelhecimento e um conhecimento com conteúdos técnicos predominantes como: envelhecimento como processo; qualidade de vida; maturidade; modificações; geração; dependência; incapacidade; doença; interação; entre outros. (Fig. 1).

O envelhecimento é objetivado em imagens dimensionadas pelas cinco classes ou categorias contemplando uma visão negativa e positiva do envelhecimento; dimensões biopsicossociais do envelhecimento; tempo de dúvidas e o envelhecimento como processo e como doença.

As imagens associadas ao envelhecimento (Fig.1) pontuam dimensões subjetivas importantes a serem consideradas pelos profissionais de saúde por ocasião do atendimento a pessoa idosa e na elaboração de estratégias para um atendimento mais efetivo em que considere a realidade social em que se encontram para trabalharem. As dimensões psicossociologias evidenciadas apontam ainda à necessidade de se considerar o idoso como coadjuvante no processo de manutenção de

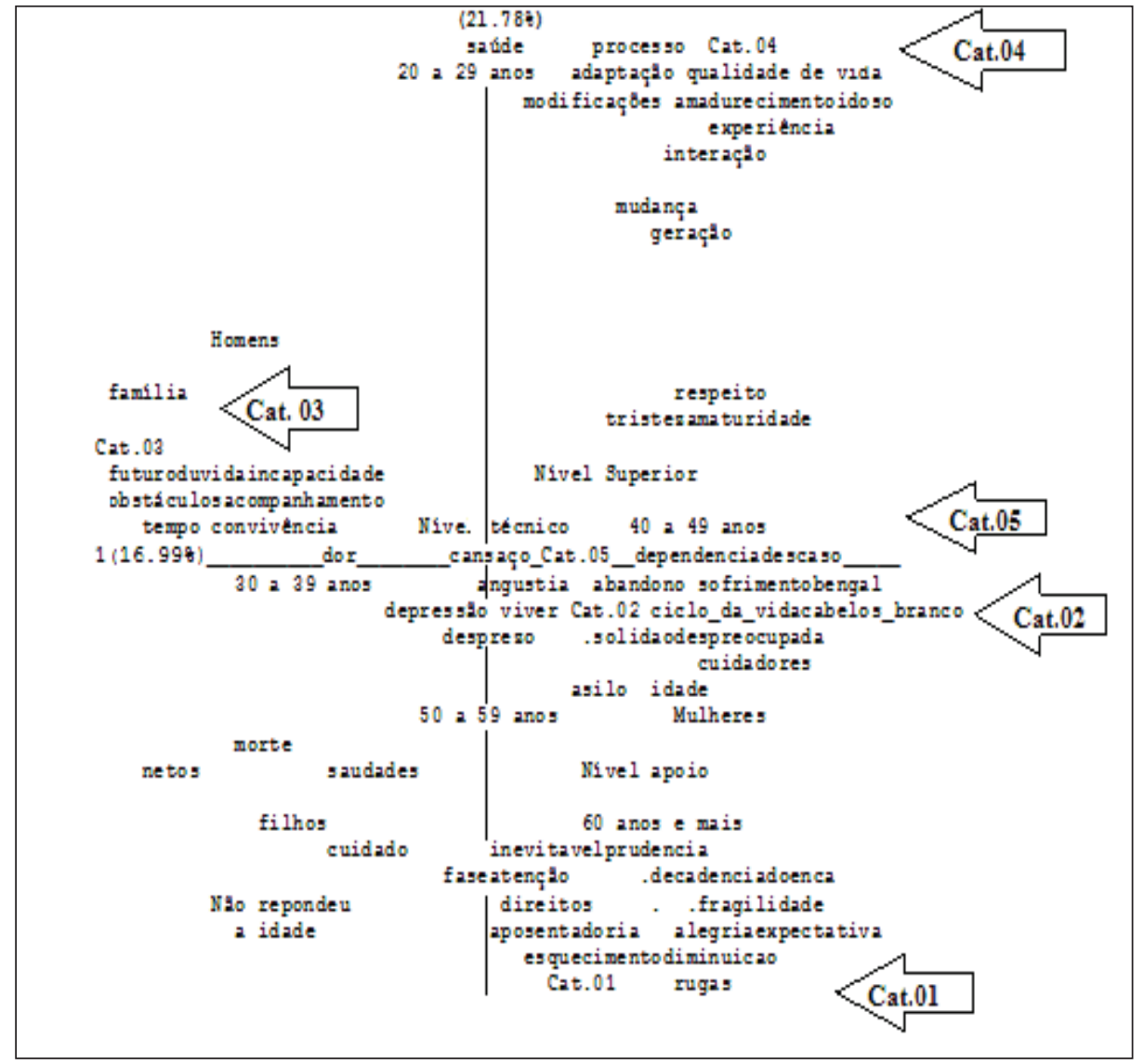

Figura 1 - Objetivação ou campo de representação do envelhecimento segundo trabalhadores de saúde. João Pessoa, PB, 2011 
sua capacidade funcional e no processo de promoção de seu bem-estar, para que possa tornar realidade à prática baseada na concepção de um envelhecimento ativo $^{(14)}$ contextualizado socialmente.

\section{CONSIDERAÇÕES FINAIS}

Este estudo procurou conhecer as representações sociais sobre envelhecimento no olhar de trabalhadores de saúde. Observou conteúdos positivos e negativos associados ao envelhecimento pelos profissionais de saúde mostrando a necessidade de um cuidar de uma forma integral e humanizada à pessoa idosa.

O envelhecimento está diretamente associado às condições e a qualidade de vida dos indivíduos. Sua avaliação para pessoa idosa implica a adoção de múltiplos critérios de natureza biológica, psicológica e socioculturais, pois vários elementos são apontados como determinantes ou indicadores de bem-estar na velhice como: longevidade, saúde biológica, saúde mental, satisfação, controle cognitivo, competência social, produtividade, atividade, eficácia cognitiva, status social, renda, continuidade de papéis familiares, ocupacionais e continuidade de relações informais com amigos ${ }^{(15)}$.

Espera-se que este estudo contribua no campo da saúde pelo seu caráter interdisciplinar em que o mesmo não se limita apenas a ela, mas a todas as áreas do conhecimento, seja pela globalização ou pela necessidade de se conhecer dimensões subjetivas sobre saúde e do processo de envelhecimento, contextualizados socialmente.

Em relação à formação do profissional de saúde, o ensino e a pesquisa têm merecido destaque, assim como, os cursos de graduação e de especialização, com um crescimento significativo, além de eventos que abordam a temática do envelhecimento, agregando profissionais com o objetivo de conscientização para a referida problemática.

As limitações da presente investigação podem ser exploradas em pesquisas futuras para um atendimento humanizado em que a conscientização da fragilidade da pessoa idosa seja considerada na atenção ao idoso como forma de minimizar os problemas próprios do envelhecimento com um pronto atendimento.

\section{REFERÊNCIAS}

1 Carvalho Filho ET, Netto MP. Geriatria: fundamentos, clínica e terapêutica. $2^{\text {a }}$ ed. São Paulo: Atheneu; 2007.

2 Moreira A, Vilar ER, Mota I, Antunes JL, Silva AC, Matos M, et al. Fórum Gulbenkian de Saúde sobre o Envelhecimento 2008/2009: o tempo da vida. Cascais: Principia; 2009.

3 Carvalho JAM, Andrade FCD. Envejecimiento de la población brasileña: oportunidades y desafíos. In: Anales del $1^{\circ}$ Encuentro Latinoamericano Y Caribeño Sobre Las Personas de Edad; 1999 sep 8-10; Santiago, Chile. Santiago: Cepal; 2000. p. 81-102. (Seminarios y Conferencias - CEPAL, 2).

4 Linck CL, Crossetti MGO. Fragilidade no idoso: o que vem sendo produzido pela enfermagem. Rev Gaúcha Enferm.2011;32(2):385-93.

5 Assis M. Promoção da Saúde e Envelhecimento: avaliação de uma experiência no ambulatório do Núcleo de Atenção ao Idoso da UnATI / UERJ [tese]. Rio de Janeiro: ENSP; 2004.

6 Mendes CKTT. Representações sociais dos trabalhadores de saúde da atenção básica dobre envelhecimento e a atendimento ao idoso (tese simplificada). Natal: Programa de Pós Graduação em Ciências da Saúde, Centro de Ciências da Saúde, Universidade Federal do Rio Grande do Norte; 2011.

7 Moscovici S. Representações sociais: investigações em psicologia social. Petrópolis, RJ: Editora Vozes; 2003.

8 Ministério da Saúde (BR), Conselho Nacional de Saúde. Resolução n. 196, de 16 de outubro de 1996: aprova as diretrizes e normas regulamentadoras de pesquisas envolvendo seres humanos [Internet]. Brasília (DF); 1996 [citado 2009 jan 20] Disponível em: http://conselho.saude.gov.br/comissao/conep/ resolucao.html

9 Moreira AOSP, Camargo BV, Jesuíno JC, Nóbrega SM. Perspectivas teórico-metodológicas em representações sociais. João Pessoa: Ed. UniversitáriaUFPB; 2005.

10 Moranas RM. Gerontologia social: envelhecimento e qualidade de vida. São Paulo: Paulinas; 2004. 
11 Papaléo Netto M. Gerontologia: a velhice e o envelhecimento em visão globalizada. São Paulo: Atheneu; 2002.

12 Ministério da Saúde (BR). Envelhecimento e saúde da pessoa idosa., Brasília(DF); 2006. (Cadernos de Atenção Básica n.19)

13 Zermerman GI. Velhice e apectos biopsicossocias. Porto Alegre: Artmed; 2000.
14 Freire Jr RC, Tavares MFL. A Saúde sob o olhar do idoso institucionalizado: Conhecendo e Valorizando sua opinião. Interface. 2005;9(16):147-158.

15 Gordilho A, Nasimento JS, Ramos LR, Freire MPA, Espinola N, Maia R, et al. Desafios a serem enfrentados no terceiro milênio pelo setor saúde na atenção integral ao idoso. Rio de Janeiro: UnATI/ UERJ; 2000.

\section{Endereço da autora / Dirección del autor /} Author's address

Cristina Katya Torres Teixeira Mendes

Rua Fernado Henrique dos Santos, 1346, ap. 402, Bessa

58037-050, João Pessoa, PB

E-mail: cristinakatya@bol.com.br

Pesquisa de Doutorado com apoio financeiro do Ministério da Saúde, 2011.
Recebido em: 02.12.2011

Aprovado em: 01.02.2012 